\title{
PHYSICAL AND NUMERICAL MODELING OF THE WAVECAT@ WAVE ENERGY CONVERTER
}

\author{
Hernán Fernández ${ }^{1}$, Gregorio Iglesias ${ }^{1}$, Rodrigo Carballo ${ }^{1}$, Alberte Castro ${ }^{1}$ and Pedro \\ Bartolomé $^{1}$
}

\begin{abstract}
Wave energy presents a great potential in many coastal regions. This paper deals with WaveCat@, a new Wave Energy Converter (WEC) recently patented by the University of Santiago de Compostela. First, the WaveCat $\odot$ concept is presented - a floating WEC intended for intermediate water depths (50-75 m), whose principle of energy capture is wave overtopping. WaveCat $\odot$ consists of two hulls, like a catamaran (hence its name); however, unlike a catamaran, the hulls are convergent so as to leave a wedge between them. Waves propagate into this wedge and, eventually, overtop the inner hull sides. Overtopping water is collected in onboard tanks and, subsequently, drained back to sea, propelling ultra-low head turbines in the process. The wave flume tests carried out on a 3D, fixed model at a 1:67 scale are presented. Development work is ongoing, including a numerical model—which is currently being validated based on the results from the physical model—and a 3D, floating physical model at a larger scale (1:30).
\end{abstract}

Keywords: wave energy; physical model; overtopping

\section{INTRODUCTION}

Given the environmental repercussions of the use of fossil fuels the share of renewable energy in the total energy production ought to be increased as fast as possible. Many regions and countries in the world have specified renewable energy targets, e.g. $20 \%$ by 2020 in the European Union, $30 \%$ by 2025 Denmark (Lund and Mathiesen 2009). In order to achieve these targets, action along two lines is necessary: in the first place, the production of the renewable energy sources that are currently exploited commercially (e.g. hydroelectric, wind, PV) should be increased; in the second place, new sources should be developed. Among the new sources, wave energy is one of the most promising. Many coastal regions have a substantial wave energy potential (e.g. Cruz 2008, Iglesias et al. 2009, Iglesias and Carballo 2009, 2010).

Wave energy may be seen as a concentrated form of wind energy in that ocean waves are generated by wind blowing over the ocean surface. Wave energy presents a number of advantages with respect to other $\mathrm{CO}_{2}$-free energy sources - high resource predictability, high power density, relatively high utilization factor and last, but not least, low environmental and visual impact relative to most alternatives. With regard to tidal energy, the other marine renewable, there exist many more potential locations for a wave farm than for a tidal energy operation, as strong tidal currents occur only in a relatively small number of areas. For these reasons, wave energy is widely regarded as one of the renewable energy sources with the greatest potential for development over the next few years.

This potential is the motivation behind the intensive efforts devoted to the development of Wave Energy Converters (WECs). WECs transform wave energy into mechanical energy and the latter into electricity. Indeed, the development of efficient, reliable WECs is a prerequisite for wave energy to become a commercially viable energy source.

WECs may be classified according to three main criteria: (i) location relative to the coastline, (ii) principle of energy capture, and (iii) wave-WEC interaction. According to their location, WECs may be: onshore, nearshore, or offshore. Onshore WECs are usually fixed structures, and their environmental impact is often nonnegligible. Nearshore WECs may be either fixed or floating structures. Offshore WECs are floating devices, and their environmental impact is usually low or very low. According to the second classification criterion, the principle of energy capture, there are three main types of WECs: Oscillating Water Columns (OWCs), Oscillating Bodies or Wave Activated Bodies (WABs), and Overtopping Devices (OTDs). In an OWC, waves cause a water column inside a chamber connected to the sea to oscillate, which in turn causes compression and depression of the air volume above the water column; these pressure variations are used to propel a Wells turbine. WABs are buoyant devices that are moved up and down by hydrostatic forces as waves advance. These oscillations are used to drive an oleohydraulic system. Finally, in OTDs waves are elevated to a reservoir above the sea level, and the energy is extracted by means of low-head turbines. Finally, the third classification criteria is wave-WEC interation. WECs may be: terminators, if the wave transmission coefficient is (practically) zero; attenuators, if there is significant wave transmission, and their size is of the order of magnitude of the wave length; and point absorbers, if there is significant wave transmission but the WEC size is small relative to the wave length.

\footnotetext{
${ }^{1}$ Hydraulic Eng., EPS, University of Santiago de Compostela, Campus Univ. s/n, Lugo, 27002, Spain
} 


\section{THE WaveCat CONCEPT}

In this context, research is under way on a number of WECs. This paper deals with WaveCat $($, a floating offshore WEC based on wave overtopping (OTD). Offshore in this context means intermediate water depths, typically in the range 50-75 m. WaveCat $\odot$ consists of two hulls, like a catamaran (hence its name); unlike a catamaran, however, the hulls are not parallel to each other but convergent (Figure 1). Waves propagate into the wedge and the convergent inner hull sides enhance wave height as waves advance. The hull freeboard is variable, decreasing towards the stern. Eventually wave crests overtop the inner hull sides and overtopping water is collected in ondeck tanks. The higher level in the tanks relative to the sea level is taken advantage of to propel ultra-low head turbines as the water is drained back to sea.

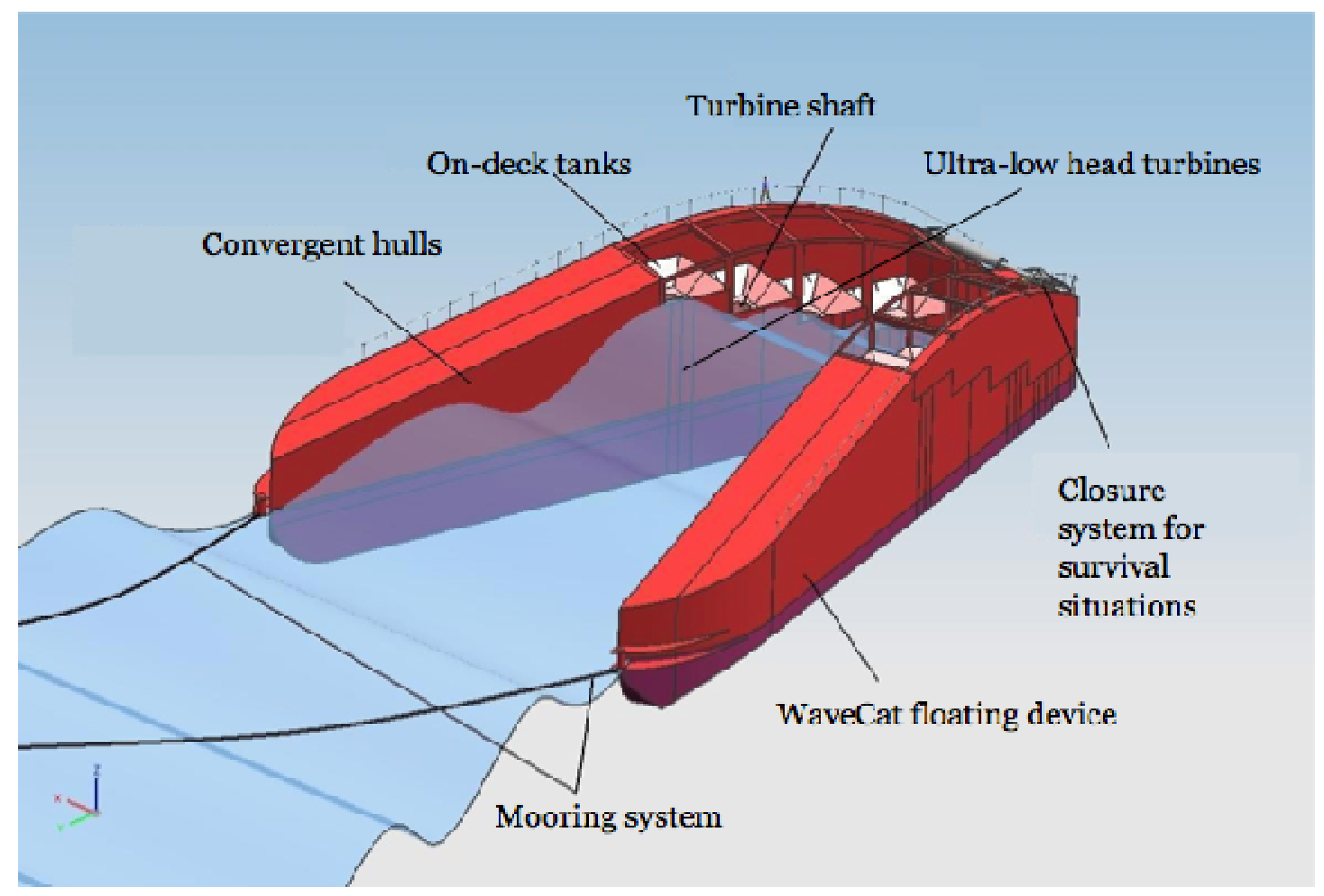

Figure 1. WaveCat@ design.

The single-point mooring system (Figures 2, 3) ensures that WaveCat swings when the wind and wave direction changes. In other words, it weathervanes around its mooring so as to stay head to sea, thereby ensuring that the incident waves propagate into the wedge formed by the convergent hulls. Given the large displacement of WaveCat, the device is moored to a catenary-buoy using a CALM configuration (Catenary Anchor Leg Mooring).

\section{PHYSICAL MODELLING}

Physical model tests were conducted in the wave flume of the University of Santiago de Compostela (Figure 4). The flume dimensions are $20 \mathrm{~m}$ (length) x $0.65 \mathrm{~m}$ (width) x $1.5 \mathrm{~m}$ (height). Wave generation is conducted by piston-type paddle. The wave generation system is equipped with the AWACS $\odot$ system for absorbing reflected waves. 


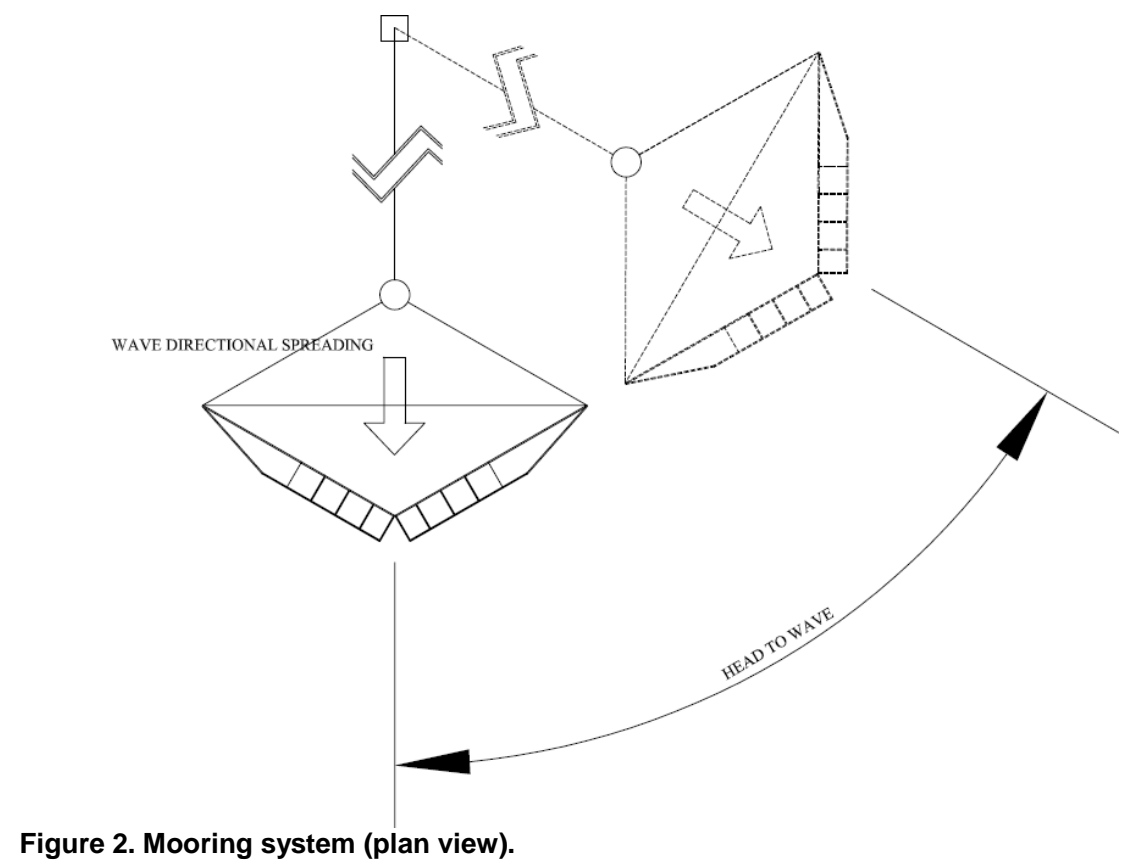

Figure 2. Mooring system (plan view).

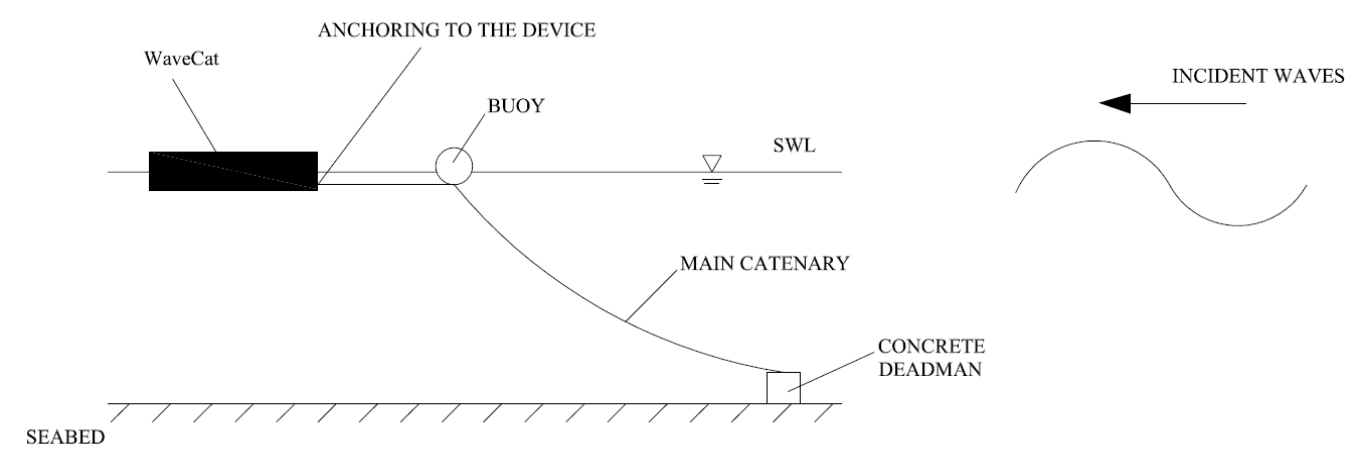

Figure 3. Mooring system with CALM configuration (vertical section).

For the physical model tests a 3D fixed model was used. Taking advantage of the design symmetry, only one of the hulls was modelled. The prototype dimensions were $90 \mathrm{~m}$ (length), $12 \mathrm{~m}$ (breadth) and 12 (hull height), and the model scale was 1:67. The physical model was constructed in methacrylate for enhanced visibility (Figure 5).

A pumping system was installed in the model (Figure 6). For reasons of space within the model, and taking into account the preliminary nature of these tests, it was decided to install a single pump rather than three, so the internal bulkheads separating the three tanks in the initial model (Figure 5) were suppressed and the model was left with a single large tank. When, in the course of a test, water in the tank reached a certain (maximum) level, the pump started automatically and emptied the tank or, to be more precise, drew water from it until a certain (minimum) level was reached. The pumping rate was calibrated prior to the actual tests. This pumping system allowed to carry out tests with a large number of waves. 


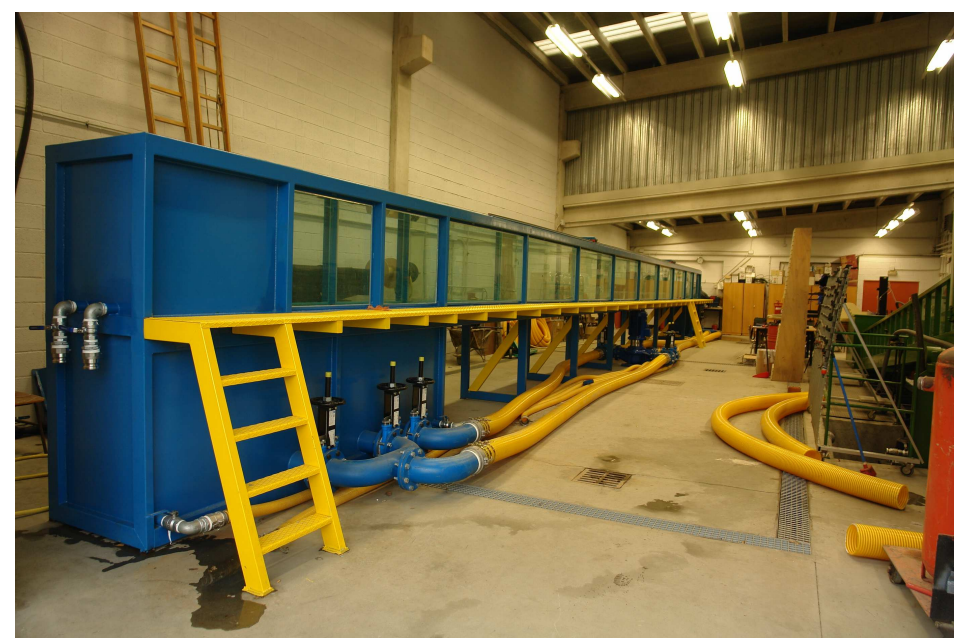

Figure 4. Wave flume at the University of Santiago de Compostela.

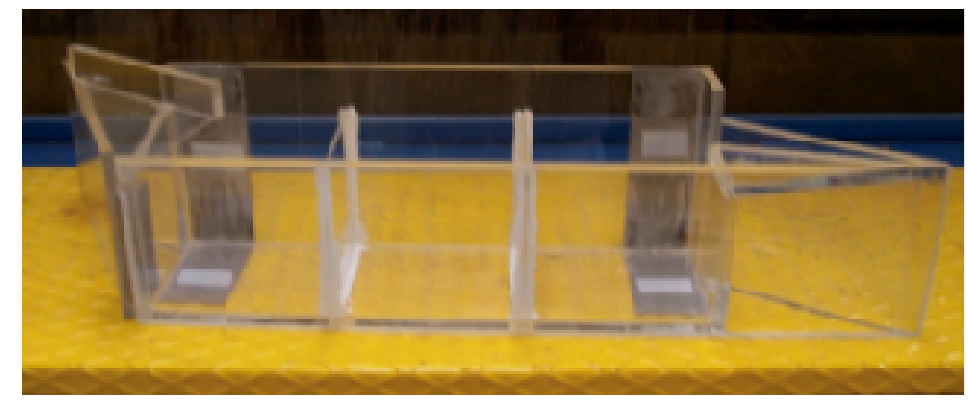

Figure 5. Methacrylate model of one hull (initial design).

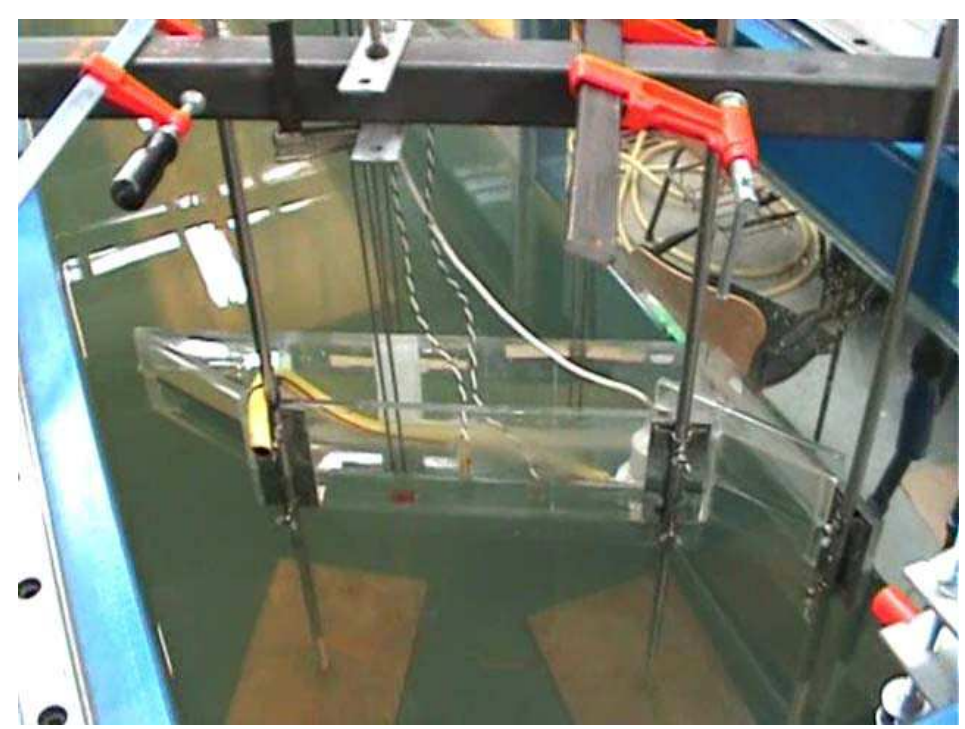

Figure 6. Physical model in the wave flume with pumping system.

The laboratory setup comprised eight wave gauges. The first four gauges (WG1, WG2, WG3, and WG4) were placed in the flume centerline. WG5 and WG6 were placed adjacent to the model front wall. WG7 measured the water level in the model tank. Finally, WG8 was located in the lee of the 
model, in order to measure wave transmission. The location of the four wave gauges WG5-WG8 is shown in Figure 7. In addition to the signals from these wave gauges and the wave paddle, the electronic signal indicating the operation or otherwise of the pumping system was available. At the downwave end of the flume a dissipating ramp with a slope of 1:10 was set up so as to minimize wave reflection (Figure 8). Prior to installing the WaveCat model in the flume, a series of tests were conducted to measure the performance of the wave dissipation ramp under different wave conditions. The reflection coefficient in terms of wave heights was in all cases under 0.1 .
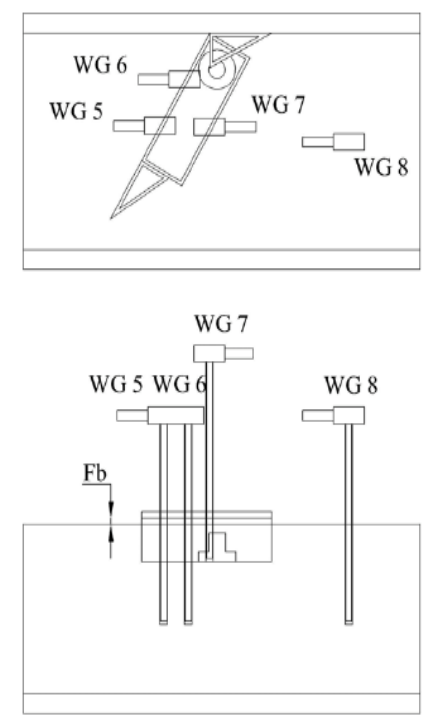

Figure 7. Experimental setup in the model area, showing the position of wave gauges WG5-WG8. [Plan view (above) and lateral perspective (below)].

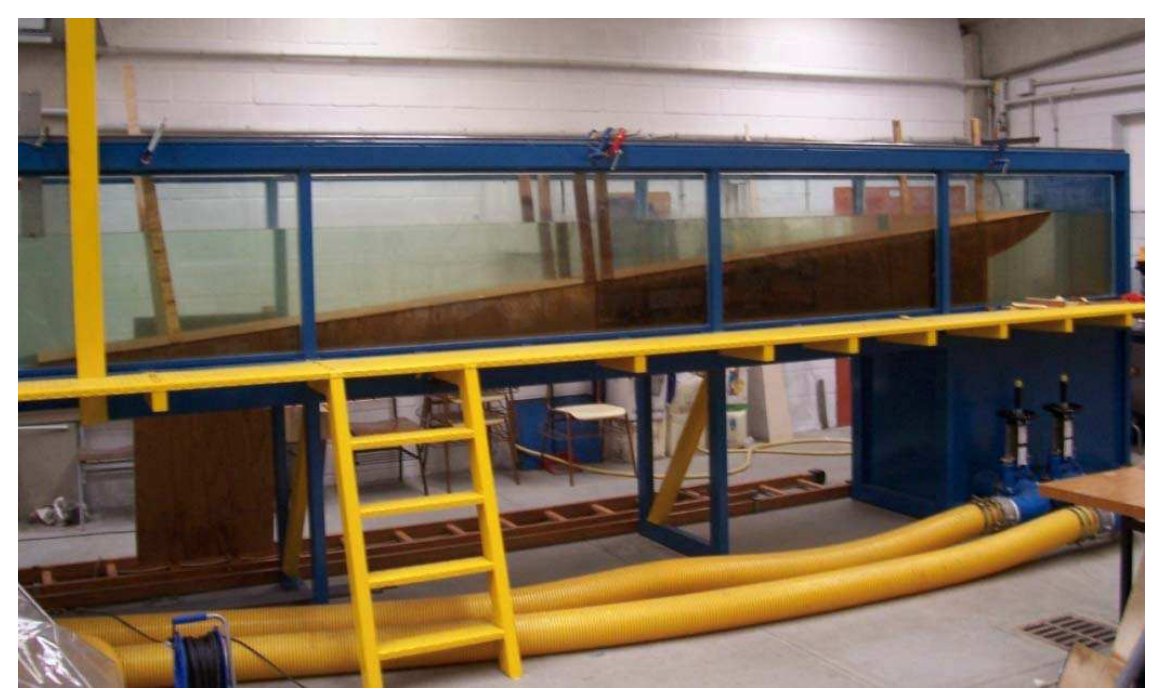

Figure 8. Wave dissipation ramp at the downwave end of the flume.

From the point of view of wave energy capture, the main parameter obtained from each test was the average overtopping rate. Based on this, other parameters could be computed: the average overtopping rate per unit length, nondimensional overtopping rate, and power. The main purpose of these physical model tests was to study the variation of the average overtopping topping for several 
wave conditions and for different values of three basic design parameters. The design parameters varied in the tests were: the freeboard, the draft, and the semi-angle of convergence between the hulls. (Naturally the first two parameters, freeboard and draft, varied concurrently). As regards the wave conditions, both regular and irregular waves were used. For regular waves, seven different wave conditions were used; wave height was varied between $2.5 \mathrm{~m}$ and $7 \mathrm{~m}$, and wave period was varied between $10 \mathrm{~s}$ and $17 \mathrm{~s}$. For irregular waves, the JONSWAP spectrum was used. In total, nine different irregular wave conditions were used. They were representative of typical sea states in Galicia, NW Spain, based on the study by Iglesias et al. (2009). The significant wave height ranged between $2 \mathrm{~m}$ and $7 \mathrm{~m}$, and the peak period varied between $10 \mathrm{~s}$ and $17 \mathrm{~s}$. For illustration, results from a regular and an irregular wave experiment are presented in Figures 9 and 10, respectively.

\section{CONCLUSIONS AND FUTURE WORK}

WaveCat $\odot$, an international patent of the University of Santiago de Compostela, is a floating WEC based on wave overtopping and intended for offshore locations (50-75 m of water depth). It presents a number of advantages relative to other WECs. First, due to its floating nature and its offshore location, its environmental impact is lower than other WEC's. Second, its moving parts are simple and wellproven: ultra-low head turbines (and the corresponding generators). This constitutes a major advantage from the point of view of reliability - a key aspect for economic viability - relative to other WECs that involve complicated mechanical parts moving with each wave. Third, with respect to other floating WECs based on wave overtopping, the major advantage of WaveCat $\odot$ is that it collects overtopping water along the inner boards of the hulls, hence along the direction of wave advance-rather than at a ramp perpendicular to the wave direction. For this reason, it may be expected that its motions as a floating body in the presence of waves (especially pitch or heave) will affect the point along the hull where overtopping begins, but not so much the overtopping volume itself as in the case of other WECs. It is expected that this advantage will be borne out for the physical and numerical experiments of a floating, 3D model to be carried out in the near future.
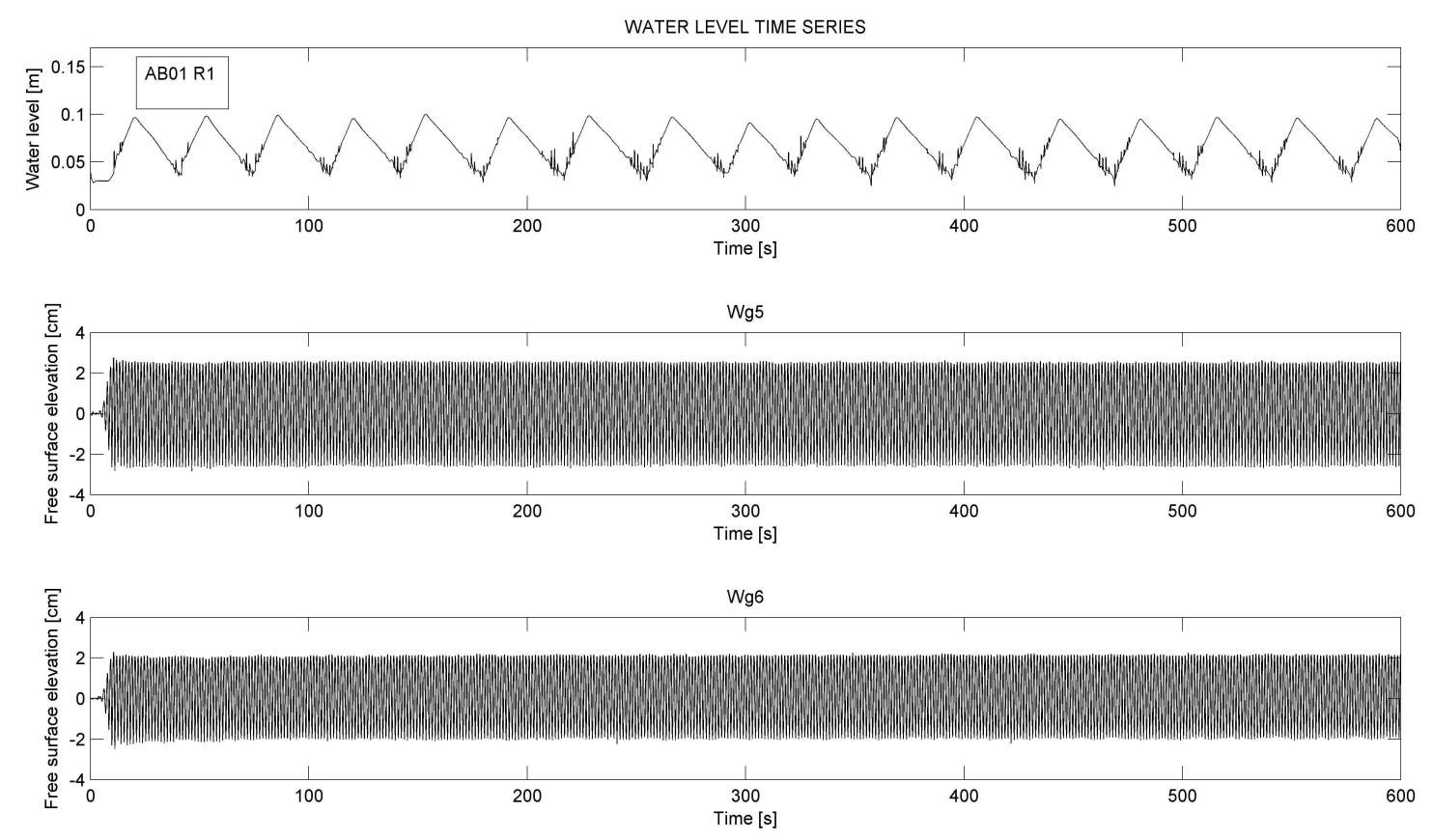

Figure 9. Water level in the tank (above, wave gauge WG7) and wave runup at the WaveCat front wall (middle and below, wave gauges WG5 and WG6, respectively) during a regular wave test. 

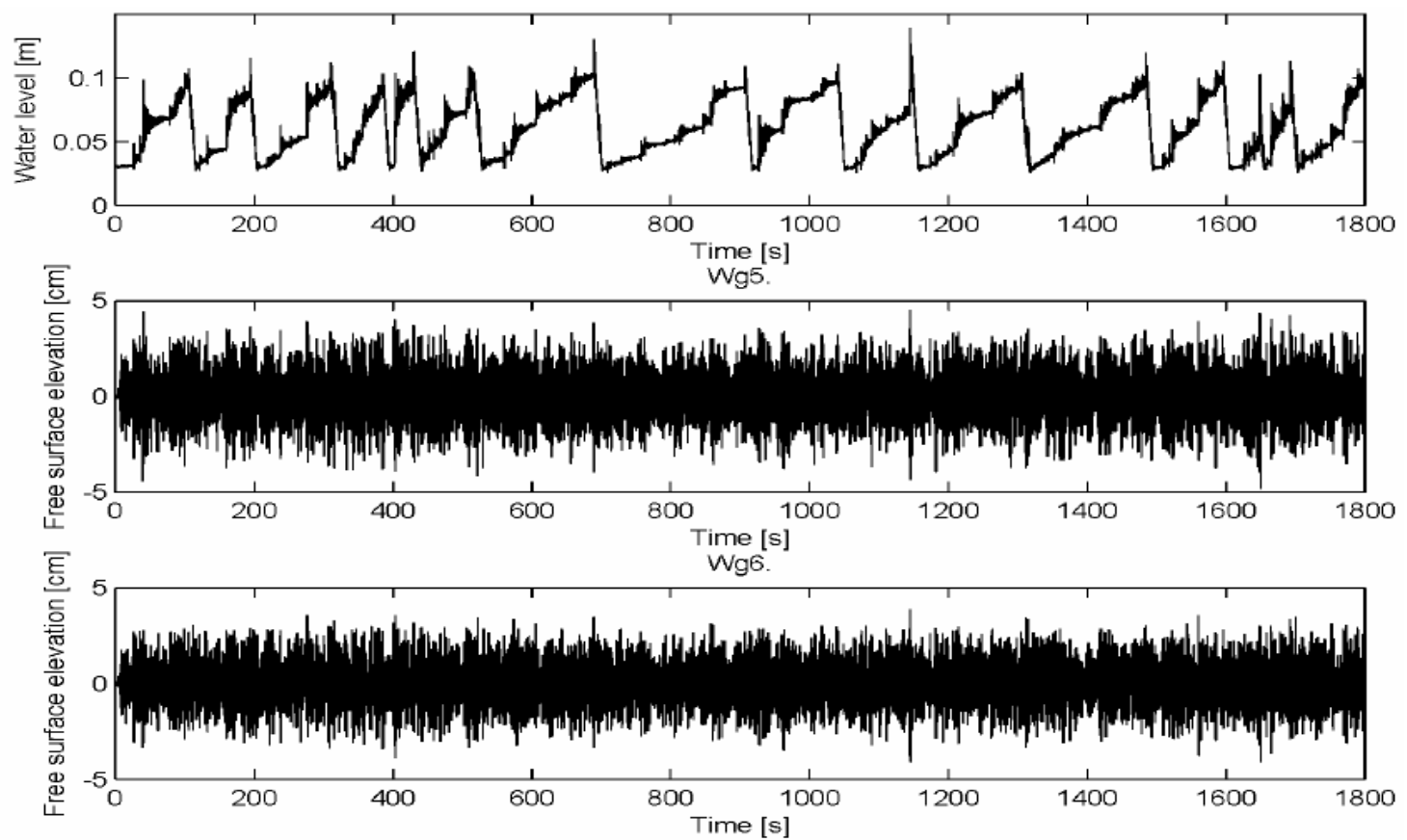

Figure 10. Water level in the tank (above, wave gauge WG7) and wave runup at the WaveCat front wall (middle and below, wave gauges WG5 and WG6, respectively) during an irregular wave test.

In this work, a 3D, fixed physical model of one of the two hulls-taking advantage of the symmetry of WaveCat $\odot$ - was constructed and tested in a wave flume under regular and irregular waves (16 different wave conditions in total). In addition to the wave conditions, three main design parameters were varied in the tests: freeboard, draft, and semi-angle of convergence.

The development of WaveCat $\odot$ is an ongoing work, with two parallel lines of action: the development of a numerical model and the preparation, realization, and analysis of physical model tests on a floating model at a larger scale (1:30). The results from the physical model tests carried out so far on the fixed model are being used for the design of a 3D, floating physical model at a larger scale (1:30), to be tested in a wave tank. In addition, the results are being used for calibrating and validating a numerical model - a Volume-of-Fluid model that solves the Reynolds-Averaged Navier-Stokes (RANS) equations.

\section{ACKNOWLEDGMENTS}

This project was funded by the Galician regional government (Xunta de Galicia) under grant 07REM001CT.

\section{REFERENCES}

Cruz, J., 2008. Ocean wave energy, Springer, Heidelberg.

Iglesias, G., López, M., Carballo, R., Castro, A., Fraguela, J.A., and Frigaard, P. 2009. Wave energy potential in Galicia (NW Spain), Renewable Energy, 34, 2323-2333.

Iglesias, G., and Carballo, R. 2009. Wave energy potential along the Death Coast (Spain), Energy, 34, 1963-1975.

Iglesias, G., and Carballo, R. 2010. Offshore and inshore wave energy assessment: Asturias (N Spain), Energy, 35, 1964-1972.

Iglesias, G., and Carballo, R. 2010. Wave energy and nearshore hot spots: The case of the SE Bay of Biscay, Renewable Energy, 35, 2490-2500.

Lund, H., and Mathiesen, B.V. 2009. Energy system analysis of 100\% renewable energy systems, The case of Denmark in years 2030 and 2050, Energy, 34, 524-531. 